BASIC RESEARCH

\title{
Cyclo-oxygenase-2 (COX-2) expression at the site of recent myocardial infarction: friend or foe?
}

\author{
A Abbate, D Santini, G G L Biondi-Zoccai, S Scarpa, F Vasaturo, G Liuzzo, R Bussani, F Silvestri, \\ F Baldi, F Crea, L M Biasucci, A Baldi
}

Heart 2004;90:440-443. doi: 10.1136/hrt.2003.010280

See end of article for authors' affiliations ......................

Correspondence to: Dr Antonio Abbate, Institute of Cardiology, Catholic University, Largo A Gemelli 8, 00168 Rome, Italy; abbatea@yahoo.com

Accepted

2 September 2003
Background: Cyclo-oxygenase-2 (COX-2) is induced in cardiomyocytes only in response to stress, such as ischaemia.

Objective: To assess COX-2 expression at the site of recent myocardial infarction.

Methods: COX-2 expression was evaluated by specific immunostaining in cardiomyocytes from 23 subjects who died 10-60 days after acute myocardial infarction. The relation between COX-2 myocardial expression and apoptotic rate was investigated. Cardiomyocyte apoptotic rate was defined as the number of cells co-expressing in situ end labelling of DNA fragmentation (TUNEL) and immunostaining for activated caspase-3.

Results: COX-2 expression was found in cardiomyocytes at the site of infarction in nine of 23 cases (39\%). It was associated with fivefold higher apoptotic rates (median 17.9\% (interquartile range 11.0-25.4\%) $v$ $3.7 \%(0.6-12.8 \%) ; p=0.016)$, and apoptotic rate increased progressively from mild to intense COX-2 staining ( $p$ for trend 0.009). COX-2 expression co-localised with TUNEL nuclear staining in myocytes, and there was a high concordance between COX-2 and hypoxia induced factor 1- $\alpha$ staining $178 \%$, $p=0.021)$ and between COX-2 and bax (83\%, $p=0.014)$. Subjects showing myocardial COX-2 expression were more likely to have enlarged hearts $(p=0.050)$, and intense COX-2 staining was strictly associated with symptomatic heart failure ( $p=0.035)$.

Conclusions: COX-2 is expressed in cardiomyocytes in nearly $40 \%$ of cases at the site of recent acute myocardial infarction, even late after the index event. Its expression was associated with extremely high apoptotic rates. These findings suggest a potential cause-effect link between COX-2 expression and enhanced myocardial apoptosis in ischaemic cardiomyopathy.

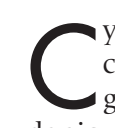
yclo-oxygenase-2 (COX-2) is one of the two isoforms of cyclo-oxygenase, the rate limiting enzyme in prostaglandin synthesis, catalysing the conversion of arachidonic acid to prostaglandin $\mathrm{H} 2$. While cyclo-oxygenase-1 (COX-1) is present in most cells and is responsible for constitutive prostaglandin formation, COX-2 is only induced in cardiomyocytes in response to stress, such as ischaemia, ${ }^{12}$ and it is therefore not expressed in healthy myocardium. Myocardial expression of $\mathrm{COX}-2$ has been reported in patients with end stage heart failure ${ }^{3}$; however, extensive data on the presence of COX-2 in cardiomyocytes after acute myocardial infarction are lacking. Moreover, while COX-2 expression has been variably associated with apoptosis in neoplastic disorders and more recently in cerebrovascular disease, there are no available data addressing this issue in the myocardium. Apoptosis may play a central part in the pathophysiology of the acute phases of myocardial infarction as well as in late postinfarction remodelling; it localises to hypoxic regions, and COX-2-which is induced in ischaemia-could have a causal role in the apoptotic cascade. ${ }^{45}$

To assess whether COX-2 expression occurs at the site of recent acute myocardial infarction and whether it is related to cardiomyocyte apoptosis, we used specific immunostaining to study its expression in cardiomyocytes at the time of necropsy from hearts of subjects who died 10 days to two months after acute myocardial infarction and who had no clinical or pathological evidence of reinfarction.

\section{METHODS}

Pathology

Twenty three consecutive cases were selected at necropsy. Sampling of the hearts was done in the peri-infarct regions as previously described. ${ }^{6}$ Briefly, a gross pathological examination was used to define the infarct area, infarct extension, infarct related artery status, gross indices of cardiac remodelling (transverse and longitudinal diameters, left ventricular free wall thickness, transverse diameter to wall thickness ratio). The clinical characteristics of each patient were obtained from the case records. Heart failure was defined as the presence of signs or symptoms of pulmonary or systemic congestion at rest, as previously described. ${ }^{7}$

COX-2 expression was evaluated using a primary antiCOX-2 antibody (goat polyclonal sc-1745, Santa Cruz Biotechnology, California, USA) at room temperature for one hour at a 1:100 dilution (according to the results of titration experiments for optimal dilutions), and a secondary reaction using the streptavidin-biotin system (Dako, Carpintera, California, USA), with diaminobenzidine as the final chromogen. Results were assessed by two independent blinded pathologists on a dicothomic (positive/negative) basis, and positive results were subsequently graded as mild $(+)$ or intense $(++)$ staining for COX-2, depending on the intensity and extension of the staining; thus a three step scale of COX-2 expression was used: none $(-)$, mild $(+)$, and intense $(++)$.

The results of the two pathologists were subsequently compared and a consensus reached in all cases. Suitable

\footnotetext{
Abbreviations: COX-2, cyclo-oxygenase-2; TUNEL, terminal deoxynucleotidyl transferase mediated dUTP-biotin $3^{\prime}-\mathrm{OH}$ nick-end labelling
} 
positive and negative controls for COX-2 expression were provided, following the manufacturer's information sheet.

From the total number of cardiomyocytes per optic field, calculated on 100 random fields, cardiomyocyte apoptotic rates were defined as the number of muscle actin positive cells co-expressing in situ end labelling of DNA fragmentation (TUNEL, Apoptag, Oncor, Gaithersburg, Maryland, USA), and immunostaining for activated caspase-3, a terminal effector of the apoptotic cascade (anti-cleaved caspase-3 antibody (Asp 175), Cell Signaling Technology, Beverly, Massachusetts, USA). Positive and negative controls for TUNEL and activated caspase-3 were provided as specified earlier. $^{6-8}$ In particular, cells expressing markers of DNA synthesis (PCNA) and/or RNA splicing (SC-35) were excluded from cell counts to avoid false positive results, and assessment of sensitivity and specificity of the assays employed for TUNEL and caspase-3 was done using human lymph nodes, as described elsewhere. ${ }^{6-8}$

As additional potential markers of ischaemia, we measured nuclear hypoxia induced factor $\alpha$ (HIF-l $\alpha$ ) staining: first, we employed mouse antihuman HIF-l $\alpha$ IgG (IgG2v, Novus Biological, Littleton, Colorado, USA) at 1:100 dilution, according to the supplier's directions, using a dicothomic positive/negative result; second, we assessed bax cytoplasmic expression (in 12 cases), using a mouse monoclonal antihuman bax sc-7480 from Santa Cruz Biotechnology, Santa Cruz, California, USA, as previously described in detail. ${ }^{6}$ Expression was graded as mild or intense, and a human lymph node was used as a control. ${ }^{6}$ HIF- $1 \alpha$ nuclear staining appears very shortly after the induction of hypoxia and disappears within 10 minutes of restoration of normal oxygen values. It therefore represents a tissue marker for hypoxia, ${ }^{9}$ and bax is a central mediator in the mitochondrial, ischaemia induced, caspase-9 dependent apoptotic cascade. ${ }^{10}$

\section{Statistical analysis}

For statistical analysis, we used SPSS 10.0 for Windows (SPSS, Chicago, Illinois, USA). Quantitative results are expressed as medians (interquartile range (IQR)). The nonparametric Kruskal-Wallis test and the Mann-Whitney U test for non-paired data were used to compare apoptotic rates among different subjects as appropriate. Logarithmic transformation was used in post-hoc testing for linear trends in univariate analysis of variance (ANOVA). Discrete variables were compared using the non-parametric $\chi^{2}$ test.

\section{RESULTS}

\section{Patient characteristics and myocardial COX-2 expression}

COX-2 expression in cardiomyocytes at the site of recent infarction was found in nine of the 23 cases (39\%). In these nine cases, COX-2 staining was observed through the entire peri-infarct region, and uniformly from the epicardial to the endocardial layer, being present in the vast majority of cardiomyocytes. COX-2 expression was mild $(+)$ in three cases $(13 \%)$ and intense $(++)$ in the remaining six cases $(26 \%)$. Figure 1 shows a case of intense COX-2 staining. The clinical characteristics of subjects did not differ between cases with COX-2 myocardial expression and the remainder. The median age of the subjects was 75 years (interquartile range (IQR) 69-81); 13 (56\%) were male, and the median time from myocardial infarction to death was 20 days (absolute range 10-62 days) (table 1).

\section{Association between myocardial COX-2 expression and apoptosis late after myocardial infarction} Overall myocardial COX-2 expression was significantly associated with a fivefold higher apoptotic rate $(17.9 \%$ (11.0-25.4\%) for peri-infarct regions with COX-2 expression,

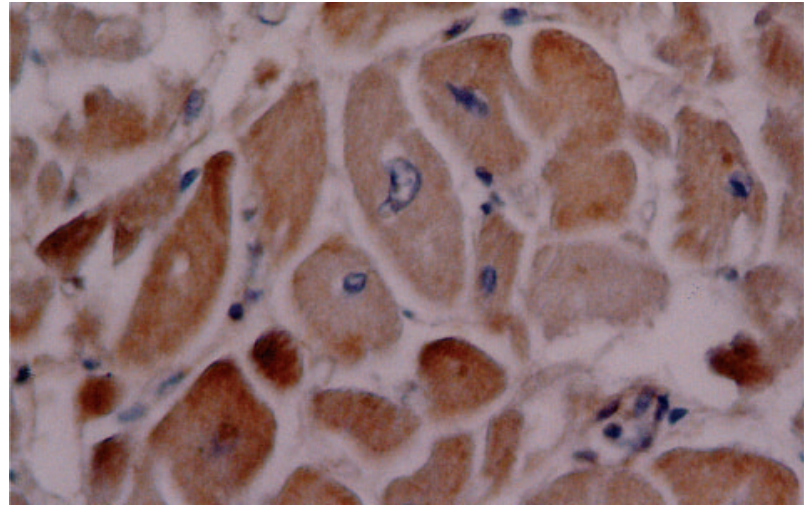

Figure 1 Intense cyclo-oxygenase-2 (COX-2) myocardial staining. Intense cytoplasmic staining is shown in several cardiomyocytes at the site of infarction, using a primary anti-COX-2 antibody (goat polyclonal sc-1745, Santa Cruz Biotechnology, California, USA, at a 1:100 dilution, according to the results of titration experiments for optimal dilutions), and with a secondary reaction using the streptavidin-biotin system (Dako, Carpintera, California, USA), with diaminobenzidine as the final chromogen.

Table 1 Characteristics of the patients according to myocardial COX-2 expression at the site of recent infarction

\begin{tabular}{lll}
\hline & COX-2 (+) & COX-2 (-) \\
\hline Number of cases (\%) & $9(39 \%)$ & $14(61 \%)$ \\
Age (years) (median (IQR)) & $74(68-84)$ & $76(69-84)$ \\
Sex (male, \%) & $6(67 \%)$ & $7(50 \%)$ \\
Clinical features & $7(78 \%)$ & $7(50 \%)$ \\
Heart failure (\%) & $2(22 \%)$ & $7(50 \%)$ \\
Anterior AMI (\%) & $3(33 \%)$ & $5(36 \%)$ \\
Previous additional AMI (\%) & $8(89 \%)$ & $13(93 \%)$ \\
Transmural AMI (\%) & $5(56 \%)$ & $9(64 \%)$ \\
Large AMI (\%) & $8(89 \%)$ & $8(57 \%)$ \\
Multivessel coronary disease (\%) & $2(22 \%)$ & $4(29 \%)$ \\
Diabetes mellitus (\%) & $7(78 \%)$ & $5(50 \%)$ \\
Permanent infarct related artery occlusion & $23(14-42)$ & $16(12-29)$ \\
Time from AMI to death (days) & $9(5-13)$ & $10(5-13)$ \\
(median (IQR)) & & \\
Norris coronary prognostic index & 9 (m) & \\
(median (IQR) & & \\
\hline
\end{tabular}

No significant differences were found when comparing the characteristics of the subjects in the two groups.

$A M I$, acute myocardial infarction; $I Q R$, interquartile range.

$v 3.7 \%(0.6-12.8 \%)$ for those without COX-2 expression; $\mathrm{p}=0.016)$. Apoptotic rates were also increasingly greater when progressing from no COX-2 expression (-) to mild (+) and intense $(++)$ expression $(3.7 \%(0.6-12.8 \%), 14.6 \%(6.4-$ $17.9 \%$ ), and $24.5 \%$ (11.5-26.7\%), respectively; p for trend 0.009 ) (figs 2 and 3). Moreover the vast majority of TUNEL+ cells in COX-2 positive cases showed co-localisation for the two markers (fig 4).

When compared with the other hearts, COX-2 positive hearts showed a greater diameter to wall thickness ratio (12.2 $(8.6-13.3) \vee 8.7(7.4-10.5), p=0.050)$, which is considered to be a marker of unfavourable postinfarction remodelling. Furthermore, subjects with symptomatic heart failure at the time of their initial hospital admission, or thereafter before death, were more likely to have intense myocardial COX-2 staining $(++)$ than the others $(p=0.035)$ (fig 5).

\section{COX-2 expression and markers of ischaemia}

The link between ischaemia, COX-2 expression, and apoptosis was strengthened by the finding of intense immunostaining 


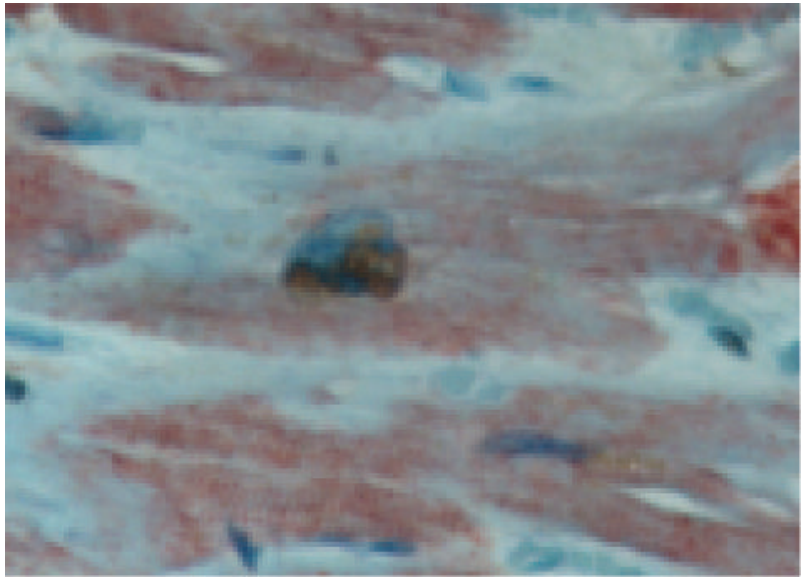

Figure 2 Cardiomyocyte apoptosis. An apoptotic cardiomyocyłe costaining for DNA fragmentation (TUNEL) and activated caspase-3 is shown.

in COX-2 positive myocardial regions for both HIF- $1 \alpha$ and bax, with a high concordance of COX-2 and HIF-1 $\alpha$ expression (74\% of cases, $\mathrm{p}=0.020)$, and of COX-2 and bax expression $(83 \%, \mathrm{p}=0.014)$.

\section{DISCUSSION}

These data, despite being limited by the small sample, confirm previous reports of COX-2 expression in ischaemic heart disease, ${ }^{3}$ and show for the first time that COX-2 expression in cardiomyocytes occurs 10 days to two months after acute myocardial infarction and is associated with an increased rate of postinfarction myocardial apoptosis. Whether this association reflects a common though independent pathway of ischaemia-driven COX-2 expression and apoptosis, or whether there is a cause-effect link between the two events, is currently unknown and cannot be determined from our data.

COX-2 expression following myocardial and cerebral ischaemia has been shown consistently in experimental models. ${ }^{21-13}$ COX-2 mRNA expression is already induced four hours after ischaemia onset, while COX-2 protein expression peaks at 24 hours. $^{2}{ }^{11-13}$ Though COX-2 expression after ischaemic insults in preconditioning animal models has been considered a protective mechanism, ${ }^{211}$ several reports have suggested that it plays a detrimental role in cerebral ischaemic damage and apoptosis. ${ }^{12-15}$ Both early and late COX-2 expression after ischaemic brain damage in rats after middle cerebral artery occlusion have been specifically investigated and shown to occur early in the ischaemic regions and late at the infarct border zones. ${ }^{12-14}$ Similarly, Takadera and colleagues have shown upregulation of COX-2 and subsequent enhanced production of COX-2 derived prostaglandin E2 in cerebral ischaemia, and an associated increase in caspase-3 dependent apoptosis. ${ }^{15}$ An alternative intriguing hypothesis, however, is to consider COX-2 expression independently from myocardial ischaemia. As an enhanced inflammatory response and neurohumoral activation have been shown to occur after acute myocardial infarction, COX-2 expression in cardiomyocytes-as in other tissues-may be induced in response to such stimuli. ${ }^{16}{ }^{17}$ Increased COX-2 synthesis may therefore be the expression of a state which itself is associated with increased apoptosis.

Independent of its causes, however, our data showing a significant association between COX-2 expression, myocardial apoptosis, and remodelling suggest that myocardial COX-2 expression in postinfarction remodelling may be detrimental. The resolution of this issue may have profound

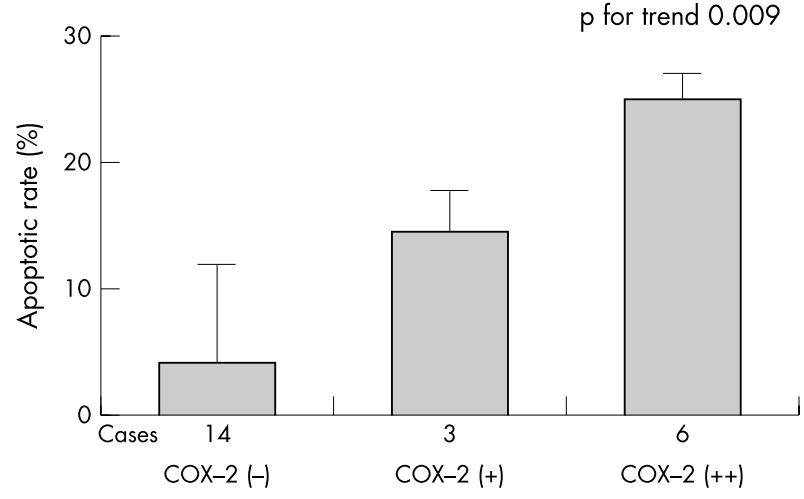

Figure 3 Peri-infarct apoptotic rate according to myocardial expression of cyclo-oxygenase-2 (COX-2). A significantly higher apoptotic rate was found in subjects with versus without COX-2 expression at the site of recent infarction, especially with intense COX-2 expression (++). Kruskal-Wallis test, $\mathrm{p}=0.047$; Mann-Whitney $\mathrm{U}$ test, $\mathrm{p}=0.016$ (comparing patients with versus without COX-2 expression); $p$ value for trend $=0.009$ (univariate ANOVA analysis considering the three groups separately). The box represents the median value and the vertical bars are the interquartile range.

clinical implications in the light of the prevalence of ischaemic heart disease, the significant role of apoptosis in progression to cardiac failure, and the widespread use of drugs acting on COX-2. Indeed, novel specific COX-2 inhibitors are currently available and may be shown to affect postinfarction cardiac remodelling. Moreover, though retrospective observational studies in humans have been inconclusive, experimental evidence shows that selective inhibition of COX-2 improves cardiac function in a rat model of acute myocardial infarction, ${ }^{18}{ }^{19}$ selective COX-2 inhibition appears to be protective against neuronal death after cerebral ischaemia, ${ }^{12}{ }^{1320}$ and in a genetically modified mouse model of non-ischaemic heart failure, COX-2 inhibition was very recently shown to be associated with improved haemodynamics and lower cardiomyocyte apoptotic rates. ${ }^{21}$

\section{Conclusions}

The inducible form of cyclo-oxygenase, COX-2, is expressed by cardiomyocytes in nearly $40 \%$ of cases at the site of acute myocardial infarction even late after the index event. The expression of COX-2 co-localises with markers of DNA fragmentation in the same myocytes at the site of the infarct,

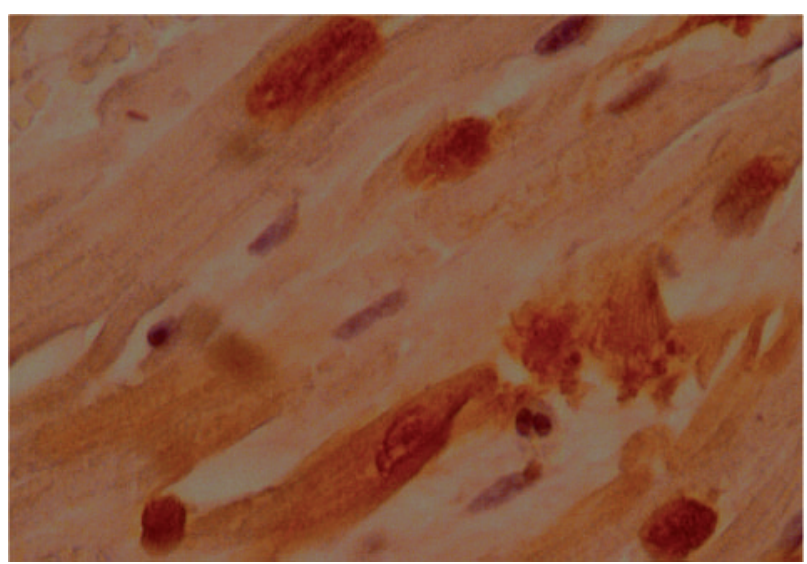

Figure 4 Cyclo-oxygenase-2 (COX-2) co-localises with markers of apoptosis. Double positive staining (nuclear staining for DNA fragmentation at TUNEL, and cytoplasmic staining for COX-2) within the same cardiomyocyte is shown. 

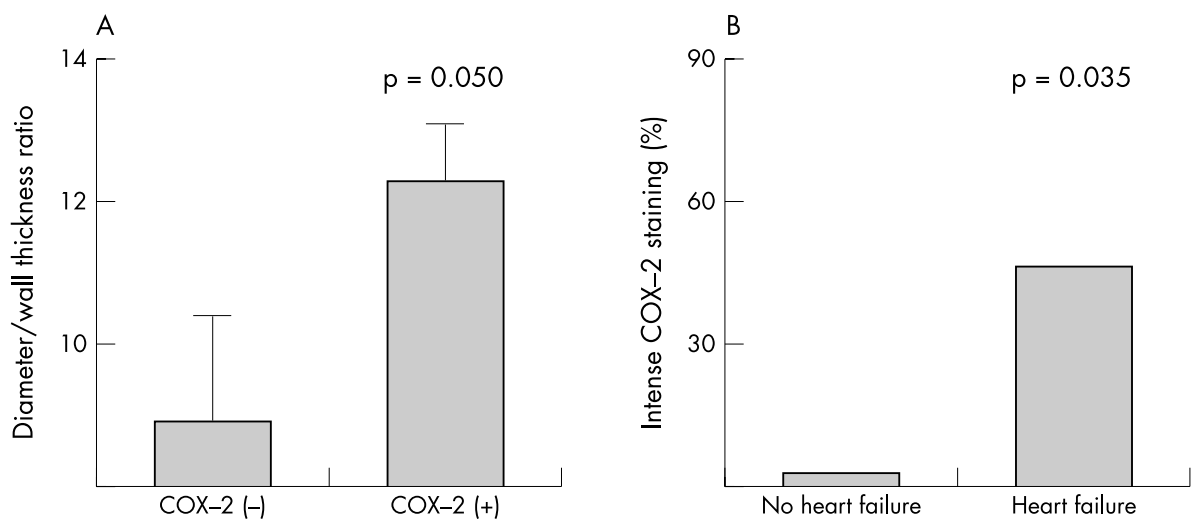

Figure 5 Cyclo-oxygenase-2 (COX-2) expression, unfavourable cardiac remodelling, and heart failure. (A) Patients with myocardial COX-2 expression at site of infarction showed a greater transverse diameter to wall thickness ratio, which is a marker of unfavourable cardiac remodelling. (B) Subjects with symptomatic heart failure were more likely to have intense periinfarct COX-2 expression than the others. The box represents the median value; vertical bars show interquartile range.

and in this necropsy model of late acute myocardial infarction is associated with extremely high apoptotic rates. These findings suggest a potential link between COX-2 expression and enhanced postinfarction myocardial apoptosis, while the presence of COX-2 in the cytoplasm of cardiomyocytes undergoing apoptosis supports an active role for COX-2 in this process.

Further studies are warranted to investigate the causal role of COX-2 in this association, to evaluate the specific role of ischaemia, inflammation, and neurohumoral activation, and to assess the potentially beneficial effect of modulating COX-2 activity.

\section{ACKNOWLEDGEMENTS}

We wish to thank Dr Vera Di Trocchio (RomaTre University of Rome, Italy) for her writing, editorial, and graphical support. This study was in part supported by a Futura Onlus grant to $\mathrm{AB}$.

\section{Authors' affiliations}

A Abbate, G G L Biondi-Zoccai, G Liuzzo, F Crea, L M Biasucci, Institute of Cardiology, Catholic University, Rome, Italy

D Santini, Section of Oncology, Campus Bio-Medico University, Rome, Italy

S Scarpa, F Vasaturo, Department of Experimental Medicine and Pathology, Università degli Studi "La Sapienza", Rome, Italy

R Bussani, F Silvestri, Department of Anatomic Pathology, University of Trieste, Trieste, Italy

F Baldi, A Baldi, Department of Biochemistry and Biophysics "F Cedrangolo", Section of Pathological Anatomy, Second University of Naples, Naples, Italy

\section{REFERENCES}

1 Smith WL, Garavito RM, De Witt DL. Prostaglandin endoperoxide $\mathrm{H}$ synthases (cyclo-oxygenases)-1 and -2. J Biol Chem 1996:271:33157-60.

2 Shinmura K, Xuan YT, Tang XL, et al. Inducible nitric oxide synthase modulates cyclooxygenase-2 activity in the heart of conscious rabbits during the late phase of ischemic preconditioning. Circ Res 2002;90:602-8.

3 Wong SCY, Fukuchi M, Melnyk $\mathrm{P}$, et al. Induction of cyclooxygenase- 2 and activation of nuclear factor-kappaB in myocardium of patients with congestive heart failure. Circulation 1998;98:100-3.
4 Mani K, Kitsis RN. Myocyłe apoptosis: programming ventricular remodeling. J Am Coll Cardiol 2003;41:761-4.

5 Bialik S, Geenen DL, Sasson IE, et al. Myocyłe apoptosis during acute myocardial infarction in the mouse localizes to hypoxic regions but occurs independently of p53. J Clin Invest 1997;100:1363-72.

6 Baldi A, Abbate A, Bussani R, et al. Apoptosis and post-infarction left ventricular remodeling. I Mol Cell Cardiol 2002;34:165-74.

7 Abbate A, Biondi-Zoccai GGL, Bussani R, et al. Increased myocardial apoptosis in patients with unfavorable left ventricular remodeling and early symptomatic post-infarction heart failure. J Am Coll Cardiol 2003;41:753-60.

8 Abbate A, Bussani R, Biondi-Zoccai GL, et al. Persistent infarct-related artery occlusion is associated with an increased myocardial apoptosis at postmortem examination in humans late after an acute myocardial infarction. Circulation 2002;106:1051-4.

9 Semenza GL. Surviving ischemia: adaptive responses mediated by hypoxiainducible factor 1. J Clin Invest 2000; 106:809-12.

10 Saikumar P, Dong Z, Patel Y, et al. Role of hypoxia-induced Bax translocation and cytochrome $c$ release in reoxygenation injury. Oncogene 1998;17:3401-15.

11 Shinmura K, Tang XL, Wang Y, et al. Cyclooxygenase-2 mediates the cardioprotective effects of the late phase of ischemic preconditioning in conscious rabbits. Proc Natl Acad Sci USA 2000;97:10197-202.

12 Nogawa S, Zhang F, Ross ME, et al. Cyclo-oxygenase-2 gene expression in neurons contributes to ischemic brain damage. J Neurosci 1997:17:2746-55

13 Nakayama M, Uchimura K, Zhu RL, et al. Cyclooxygenase-2 inhibition prevents delayed death of CA1 hippocampal neurons following global ischemia. Proc Natl Acad Sci USA 1998:95:10954-9.

14 Collaco-Moraes Y, Aspey B, Harrison M, et al. Cyclo-oxygenase-2 messenger RNA induction in focal cerebral ischemia. I Cereb Blood Flow Metab 1996;16:1366-72.

15 Takadera T, Yumoto H, Tozuka Y, et al. Prostaglandin E(2) induces caspasedependent apoptosis in rat cortical cells. Neurosci Lett 2002;317:61-4.

16 LaPointe MC, Isenovic E. Interleukin-1 beta regulation of inducible nitric oxide synthase and cyclooxygenase- 2 involves the p42/44 and p38 MAPK signaling pathways in cardiac myocytes. Hypertension 1999;33:276-82.

17 Rocha R, Rudolph AE, Frierdich GE, et al. Aldosterone induces a vascular inflammatory phenotype in the rat heart. Am J Physiol Heart Circ Physiol 2002;283: H1802-10.

18 Saito T, Rodger IW, Hu F, et al. Inhibition of cyclooxygenase-2 improves cardiac function in myocardial infarction. Biochem Biophys Res Commun 2000;273:772-5.

19 Saito T, Rodger IW, Shennib H, et al. Cyclooxygenase-2 (COX-2) in acute myocardial infarction: cellular expression and use of selective COX-2 inhibitor. Can J Physiol Pharmacol 2003;81:114-19.

20 Govoni S, Masoero E, Favalli L, et al. The cycloxygenase-2 inhibitor SC58236 is neuroprotective in an in vivo model of focal ischemia in the rat. Neurosci Lett 2001:303:91-4.

21 Zhang Z, Vezza R, Plappert T, et al. COX-2 dependent cardiac failure in Gh/ †TG transgenic mice. Circ Res 2003;92:1153-61. 